\title{
Surface Morphology of GaN: Flat versus Vicinal Surfaces
}

\author{
M.H. Xie, S.M. Seutter, L.X. Zheng, S.H. Cheung, Y.F. Ng, Huasheng Wu, S.Y. Tong \\ Department of Physics, The University of Hong Kong, Pokfulam Road, Hong Kong
}

\begin{abstract}
The surface morphology of GaN films grown by molecular beam epitaxy (MBE) is investigated by scanning tunneling microscopy (STM). A comparison is made between flat and vicinal surfaces. The wurtzite structure of GaN leads to special morphological features such as step pairing and triangularly shaped islands. Spiral mounds due to growth at screw threading dislocations are dominant on flat surfaces, whereas for vicinal $\mathrm{GaN}$, the surfaces show no spiral mound but evenly spaced steps. This observation suggests an effective suppression of screw threading dislocations in the vicinal films. This finding is confirmed by transmission electron microscopy (TEM) studies. Continued growth of the vicinal surface leads to step bunching that is attributed to the effect of electromigration.
\end{abstract}

\section{INTRODUCTION}

Current intensive experimentation on III-V nitrides has led to rapid progress in blue light and high power device applications [1]. These achievements contrast greatly an apparent lack of good understanding of many fundamental issues related to growth and film properties of nitrides. To further improve the quality of epitaxial thin films and hereafter the performance of nitride-based optoelectronic and microelectronic devices, a better knowledge of its growth kinetics and surface dynamics is obviously needed.

In a non-equilibrium growth system such as molecular beam epitaxy (MBE), surface morphology contains important kinetic and dynamic information related to the growth process [2]. Therefore, by studying the morphological evolution of surfaces during MBE growth under various conditions, key kinetic parameters governing growth can be derived.

This paper presents recent observations of many novel morphologies during growth of $\mathrm{GaN}(0001)$ on $\mathrm{SiC}(0001)$ substrates without the use of buffer layers. Both nominally flat and vicinal substrates are used. In addition to the strong anisotropy in growth rates of two types of surface steps, step bunching during vicinal film growth is also observed. Furthermore, for a vicinal film, there is no spiral mound, which is the dominant feature for a flat surface.

\section{EXPERIMENTS}

MBE growth and scanning tunneling microscopy (STM) experiments of GaN films are conducted in a multi-purpose ultrahigh vacuum (UHV) system, with background pressures in the range of $10^{-11} \mathrm{mbar}$. The MBE system is equipped with a conventional effusion cell for gallium $(\mathrm{Ga})$ and a radio-frequency plasma generator for $\mathrm{N}_{2}$. For all the $\mathrm{GaN}$ growth reported in the paper, the Ga source temperature is set at $980^{\circ} \mathrm{C}$, and the $\mathrm{N}_{2}$ flow rate at $0.13 \mathrm{sccm}$ (standard cubic centimeter per minute). The power of the plasma unit is set at $500 \mathrm{~W}$. These conditions correspond to an III/ $\mathrm{V}$ flux ratio of approximately 2 and a GaN growth rate of $0.26 \AA \mathrm{s}^{-1}$ [3]. The substrates are nominally flat $6 \mathrm{H}-\mathrm{SiC}(0001)$ 
(Cree Research, Inc) and wiona $4 \mathrm{H}-\mathrm{SWC}(000)$ ) (Nwpon Steel Co.) misoriented towards [-1010] by $3.5^{\circ}$. Pron to GaN deposition, the substrates are deoxidzed at $100 \mathrm{C}$ in a $\mathrm{S}$ fux suppled from an $e$-beam evaporatow The chemical nuty of he reated surface is monitored by in situ X-ray photoelectron spectroscopy (XPS), white the atomic order of the surface is analyzed by in situ low energy electron diffraction (LEED). Under a properly chosen $S 1$ tux condition, the SiC suraces show the $(\sqrt{3} \times \sqrt{3})$ R $30^{\circ}$. surface reconstruction following the deoxidization process. STM images of the surface show atomicaly tat teraces of more than $1000 \mathrm{~A}$ wide for the that substrate and a step-terace morphology for the vicinal substrate. GuN growth is initiated by simultateously opening the somee shuters of Ga and $\mathrm{N}$ and at substrate temperatwes berween $500 \mathrm{C}$ and $650^{\circ} \mathrm{C}$. Sample heating is achieved by passing through it a direct current $(\mathrm{OC}$ ) along the long side of the rectangular substrate pieces $\left(11 \times 4 \mathrm{~mm}^{2}\right.$ in size).

To terninate growh, the source shuters are closed simultaneously and at the sane time, the power of the plasma unit is switched off. Meanwhile, the sample is quenched rapidy by switching of the DC heating power. Due to the small sample size, he temperature drops quickly to below $400^{\circ} \mathrm{C}$ within seconds. The grenched surtace is subsequenty examined by in situ STM. Constant current mode STM experiments are conducted at room temperature at a tuneling current of $0.1 \mathrm{AA}$ and sample bias of $-3 \mathrm{~V}$.

\section{REULTS AND DISCUSSIONS}

GaN growth on both hat and vicinal swbstrates commences by hree-dimensional (3D) island formation as indicated by relection highenergy electron diffaction (RMEED) pattems. This is followed by a transition to two-dinensional (2D) growth after coalescence of $3 \mathrm{D}$ islands. It is noted that such trancition from $3 \mathrm{D}$ to $2 \mathrm{D}$ growth is sooner for the vicinal fim than lat ones. Contined growth on the two substrates leads to completely different norphologies, which is the subject of the following discussion.

\section{Flat Surrace}

Fig. 1(a) shows a GaN surace following lum deposition at $650^{\circ} \mathrm{C}$. The main observation is the dominance of spiral mounds on the suxface. The density of such mounds is estimated from the inage to be $2 \times 10^{9} \mathrm{~cm}^{2}$. As growth spinals originate from screw or mixed-type theading dislocations in the fum $[A]$, it is conduded that the densty
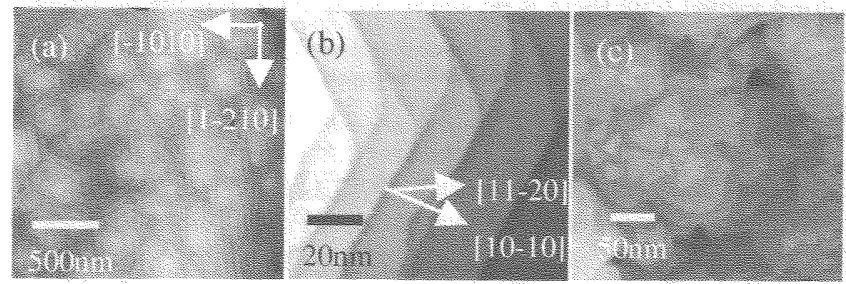

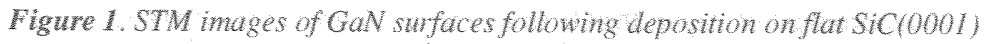
subrtates at (a) and $(b) 650^{\circ} \mathrm{C}$. (o) $500^{\circ} \mathrm{C}$. Now the scale diference benen (a) and $(b)$. 
of screw threading dislocations in the flat film is of the order of $2 \times 10^{9} \mathrm{~cm}^{-2}$, which has been confirmed by transmission electron microscopy (TEM) investigations [5]. Similar morphologies have also been reported by others [6,7], therefore, it seems that it is characteristic to MBE-grown GaN films on flat substrates. Note that if a pair of growth spirals originates from growth at two vertical screw arms of a ' $U$ '-shaped dislocation line, then the spirals in the pair ought to have opposite rotation direction. Indeed, both clockwise and anti-clockwise rotation spirals are seen.

Zooming-in one of the spiral mounds reveals a special step structure, as shown in fig. 1 (b). Along one of the $<10-10\rangle$ crystallographic directions, steps are all double bilayer (BL) high, i.e., it equals $c=5.2 \AA$, the height of the unit cell of GaN in the [0001] direction. In between the two adjacent double BL steps, along the [11-20] direction, the steps are de-paired into single BL and a twilled structure results. Such a step structure is understood by considering the dangling bond characteristics of a wurtzite film [8]. For such a film's surface, there are two types of steps, namely type-A and type-B steps. For a type-A step, each edge atom has two dangling bonds whereas for type-B steps, the edge atom has only one dangling bond. Therefore, for a type-A step, there is a high density of kinks whose advance is limited by the arrival rate of adatoms by diffusion. On the other hand, for a type-B step, there are few kinks, as the creation of a kink site on such a step will expose the high-energy type-A step. As a result, the motion of a type-B step may involve nucleation of one-dimensional (1D) islands along the step edge, therefore, its speed of growth is reduced compared to the growth of a type-A step. For a wurtzite film, consecutive steps along a given $\langle 10-10\rangle$ direction belong to type- $A$ and type- $B$ alternately, therefore, the fast-growing type-A step will ultimately catch up with the slower-growing type-B step underneath, and step-pairing occurs. On the same terrace, a step changes its character from type-A to type-B or vice versa upon turning $60^{\circ}$. This explains the formation of the twilled structure of single BL steps along the [11-20] direction.

Under the island nucleation growth mode at low substrate temperatures, e.g., $500^{\circ} \mathrm{C}$, 2D single BL height islands form on terraces that show triangular shape, as depicted in fig. 1(c). The triangular shape is again due to the growth anisotropy of GaN. The fastgrowing type-A step ultimately makes this step disappear and the island becomes bounded by the slower-growing, low energy, type-B steps. Unexpectedly, from the image of fig. I(c), triangular islands oriented $180^{\circ}$ with respect to each other on the same terrace are seen. To explain this, we assume atoms in some of the islands are wrongly stacked. For example, instead of the wurtzite ABAB... stacking, atoms take the cubic ABCA... stacking instead. The oppositely oriented triangular islands can also originate from twins in a pure cubic film, i.e., one with $\mathrm{ABCA}$... and the other with ACBA... stacking. It has been shown by TEM studies that low growth temperature promotes cubic film formation and there is a high density of stacking faults and twin boundaries in such a film [9]. Therefore, the assertion of the $180^{\circ}$-oriented islands to stacking faults and twins is likely to be correct.

\section{Vicinal Surface}

Fig. 2 shows a GaN surface following a $0.5 \mu \mathrm{m}$ film deposition at $650^{\circ} \mathrm{C}$ on a vicinal $\mathrm{SiC}$ substrate. The most remarkable difference between this surface and the flat one (fig. $1(\mathrm{a}))$ is the absence of spiral mounds. As described earlier, spiral mounds reflect growth at screw threading dislocations. Therefore, the absence of spirals in fig. 2 implies a 
Figure 2. STM image of a GaN film grown on a vicinal $4 \mathrm{H}$-SiC(0001) substrate at $650^{\circ} \mathrm{C}$. Note the absence of spiral mounds.

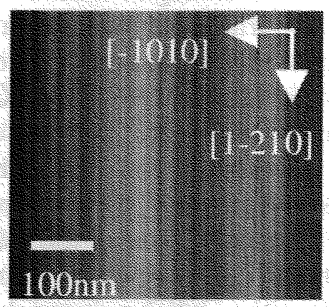

suppression of threading screw dislocations in the vicinal film. This suggestion has been confirmed by TEM studies, which reveal a density of screw threading dislocation in the vicinal film of the order of $10^{7} \mathrm{~cm}^{-2}$. This is two-orders of magnitude less than that in the flat film. In addition to screws, TEM images also suggest a reduction of threading edge dislocations. Therefore, judging by these results, the quality of the vicinal epitaxial film is substantially improved. The cause of dislocation suppression is still under investigation, preliminary results indicate that it is likely related to the initiation and coalescence processes of islands when $\mathrm{GaN}$ is initially grown on $\mathrm{SiC}$. Examining the individual steps reveals they are all double $B L$ high, in agreement with the growth anisotropy described earlier.

Let's now consider the implication of the above step structure to step kineties during MBE growth. Referring to the ID model of a vicinal GaN surface as shown in fig. $3, X_{A}$ and $X_{B}$ denote the positions of steps $A$ and $B$ belonging to type-A and type-B, respectively. Let $W$ be the terrace width bounded by the two steps, while $W_{+}$and $W$ are the terrace widths in front of and behind the $B$ and $A$ steps, respectively (see fig. 3). If $k_{A, B}^{ \pm}$are adatom incorporation coefficients to steps from their respective upper (-) and lower $(+)$ terraces, then the speeds of motion of the steps under the step-flow growth mode can be written as [10]:

$$
\begin{aligned}
& \frac{\partial X_{A}}{\partial t}=k_{A} W_{-}+k_{A}^{+} W \\
& \frac{\partial X_{B}}{\partial t}=k_{B} W+k_{B}^{+} W_{+}
\end{aligned}
$$

In writing down equ. (1), we have ignored the lateral fluctuations of steps. The two terms on the right-hand side of the equation reflect contributions from the upper and lower terraces, respectively. Subtract the two equations and note that $W=X_{B}-X_{A}$, one obtains

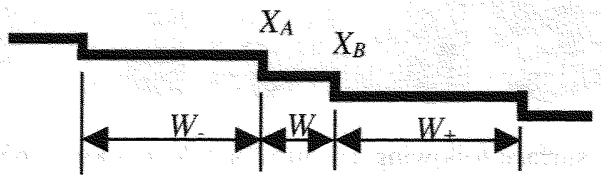

Figure 3. A $1 D$ model of a vicinal GaN surface. The consecutive steps belong to twpe-A and twpe-B, respectively, as required by the wurtzite crystal structure. 


$$
\begin{aligned}
\frac{\partial W}{\partial t} & =k_{B}^{+} W_{+}-k_{A}^{-} W_{-}+\left(k_{B}^{-}-k_{A}^{+}\right) W \\
& \approx k_{B}^{+} W_{+}-k_{A}^{-} W_{-}
\end{aligned}
$$

where we have used an approximation of $W \approx 0$ in deriving the second equality. It is valid when step-pairing has occurred (i.e., $A$ step has caught up with the $B$ step) and so there is no terrace between the two steps. Note further that statistically, $W_{+}=W_{\text {, as }}$ as tho terraces are equivalent. Therefore,

$$
\frac{\partial W}{\partial t} \propto k_{B}^{+}-k_{A}^{-}
$$

In order to maintain the double BL step (DBS) structure during growth, one requires $\frac{\partial W}{\partial t} \leq 0$, which leads to $k_{B}^{+} \leq k_{A}^{-}$. This mean that adatom capture by a type-A step from its upper terrace $\left(k_{A}^{-}\right)$is at least as efficient as adatom capture by a type-B step from its lower terrace $\left(k_{B}^{+}\right)$.

Once a DBS has formed, which constitutes an $A$ step on top and a $B$ step below, the motion of such a DBS 'unit' is determined by adatom incorporation from the upper terrace behind step $A$ and from the lower terrace in front of step $B$. If, as argued above, the rate of incorporation of adatoms from the upper terrace is higher than that from the lower terrace, continued growth will lead to further step bunching [10]. A complication comes, however, from the fact that adatom incorporation at a DBS may be different than that of an adatom diffusing down a step. At a DBS, adatoms need to cascade two BL height steps, $A$ and $B$, in order to be successfully incorporated in the step. On the other hand, step bunching following continued growth of the vicinal film has indeed been observed [11]. An example of a bunched surface is shown in fig. 4, which is obtained after GaN deposition for another $2.5 \mu \mathrm{m}$ on top of the surface given in fig. 2 . It is further shown that the bunching behavior depends on the DC direction that is applied across the sample in order to maintain the substrate temperature. Step bunching occurs only when the electric field points to the step down direction of the vicinal surface. When the field is reversed, the bunched steps tend to debunch. This observation, therefore, points to the electromigration effect $[11,12]$. If so, according to the field dependence, we infer that adatoms, which are likely $\mathrm{N}$ in this case when the growth is under Ga-rich condition, possess effective positive charges. At the moment, it is not clear whether step bunching is

Figure 4. STM image showing step bunching of the vicinal GaN film following prolonged growth at $650^{\circ} \mathrm{C}$ and heated by a DC along the step -down direction (from left to right).

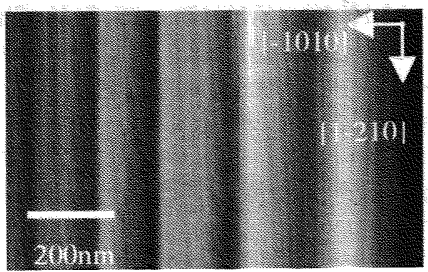


caused solely by the electromigration effect, or it is also caused by a higher rate of incorporation of adatoms from the upper terrace compared to that from the lower terrace. More studies are needed to separate out these two effects.

\section{CONCLUSIONS}

We have summarized our observations of surface morphology of $\mathrm{GaN}$ during its $\mathrm{MBE}$ growth. A comparison is made between the flat and vicinal surfaces, where the former shows spirals mounds due to screw threading dislocations, contrasting to vicinal films where there is no spiral mound. This suggests an effective suppression of threading dislocations. Furthermore, strong growth anisotropy and step bunching are observed, which can have important implications in the design of nitride-based device structures.

\section{ACKNOLEDGEMENTS}

This work is supported in part by HK RGC grants No. HKU7118/98P, 7117/98P, 7142/99P and 260/95P, US DOE grant No. DE-FG02-84ER 45076, US NSF grant No. DMR-9972958. The vicinal $4 \mathrm{H}-\mathrm{SiC}$ substrate is kindly provided by Dr. N. Ohtani of Nippon Steel Corporation, Japan.

\section{REFERENCES}

1. S. Strite and H. Morkoc, J. Vac. Sci. Technol., B10, 1237 (1992)

2. Z. Zhang and M. G. Lagally (eds.), Morphological Organization in Epitavial Growth and Removal, World Scientific, Singapore, 1998

3. S. M. Seutter, M. H. Xie, W. K. Zhu, L. X. Zheng, Huasheng Wu, and S. Y. Tong, Surf. Sci. Lett.,(in press)

4. W. K. Burton, N. Cabrera, and F. C. Frank, Phil. Trans. Roy. Soc., 243, 299 (1951)

5. M. H. Xie, L. X. Zheng, Y. F. Ng, Huasheng Wu, N. Ohtani, and S. Y. Tong, unpublished

6. B. Heying, E. J. Tarsa, C. R. Elsass, P. Fini, S. P. DenBaars and J. S. Speck, J. Appl. Phys., 85, 6470 (1999)

7. A. R. Smith, R. M. feenstra, D. W. Greve, M. S. Shin, M. Skowronski, J. Neugebauer and J. E. Northrup, J. Vac. Sci. Technol., B16, 2242 (1998)

8. M. H. Xie, S. M. Seutter, W. K. Zhu, L. X. Zheng, Huasheng Wu, and S. Y. Tong, Phys. Rev. Lett., 82, 2749 (1999)

9. M. H. Xie, L. X. Zheng, S. Y. Tong, unpublished

10. P. Bennema, and G. H. Gilmer, in Crystal Growth: An Introduction (ed. P. Hartman), North-Holland, 1973, p263

11. M.H. Xie, S.H. Cheung, L.X. Zheng, Y.F. Ng, Huasheng Wu, N. Ohtani, and S.Y. Tong, unpublished

12. A. V. Latyshev, A. L. Aseev, A. B. Krasilnikov, and S. I. Stenin, Suff. Sci., 213, 157(1989) 